\title{
On perturbations in Horndeski theories
}

\author{
Victoria Volkova ${ }^{1,2, a}$ \\ ${ }^{1}$ Institute for Nuclear Research of the Russian Academy of Sciences, 60th October Anniversary Prospect, \\ 7a, 117312 Moscow, Russia \\ ${ }^{2}$ Department of Particle Physics and Cosmology, Physics Faculty, M.V. Lomonosov Moscow State University, \\ Vorobjevy Gory, 119991 Moscow, Russia
}

\begin{abstract}
This research has been carried out in collaboration with R. Kolevatov, S. Mironov, V. Rubakov and N. Sukhov. We study the approach suggested by Deffayet et al. for obtaining a derivative part of the quadratic action for scalar perturbations in the cubic Horndeski theory. We analyse the validity of the approach and generalize it for the complete Horndeski theory. We explicitly check that the generalized method gives the correct result.
\end{abstract}

\section{Introduction and summary}

The theory of generalized Galileons (or, equivalently, Horndeski theory) has acquired significant interest lately due to the search for healthy ways of violating the null energy condition (NEC). Generalized Galileons are scalar fields whose Lagrangian involves second derivatives, but the corresponding equations of motion are still second-order and, thus, are free of Ostrogradsky instabilities [1] (for a review see, e.g., Ref.[2]).

The most general Lagrangian for the Horndeski theory reads (mostly negative signature):

$$
\mathcal{L}=\mathcal{L}_{3}+\mathcal{L}_{4}+\mathcal{L}_{5}
$$

where

$$
\begin{aligned}
& \mathcal{L}_{3}=F(\pi, X)+K(\pi, X) \square \pi, \\
& \mathcal{L}_{4}=-G_{4}(\pi, X) R+2 G_{4 X}(\pi, X)\left[(\square \pi)^{2}-\pi_{; \mu v} \pi^{; \mu v}\right], \\
& \mathcal{L}_{5}=G_{5}(\pi, X) G^{\mu v} \pi_{; \mu v}+\frac{1}{3} G_{5 X}\left[(\square \pi)^{3}-3 \square \pi \pi_{; \mu v} \pi^{; \mu v}+2 \pi_{; \mu v} \pi^{; \mu \rho} \pi_{; \rho}^{v}\right] .
\end{aligned}
$$

Here $\pi$ is the Galileon field, $X=g^{\mu \nu} \nabla_{\mu} \pi \nabla_{\nu} \pi, \pi_{, \mu}=\partial_{\mu} \pi, \pi_{; \mu \nu}=\nabla_{v} \nabla_{\mu} \pi, \square \pi=g^{\mu v} \nabla_{\nu} \nabla_{\mu} \pi, G_{4 X}=$ $\partial G_{4} / \partial X$, etc.

Horndeski theories provide means for constructing the unconventional cosmological solutions, such as bouncing Universe and Genesis for instance, which are alternative or complementary to the inflationary scenario. There are numerous sound explicit examples of these solutions, constructed

\footnotetext{
ae-mail: volkova.viktoriya@physics.msu.ru
} 
within the Horndeski theory [3-10]. The central issue, however, is stability of the obtained solutions, i.e. the absence of different kinds of instabilities (ghosts, gradient instabilities, etc.).

Instabilities, if any, develop at the level of small perturbations about the background solution. In this paper we study generalized Galileons in a spatially flat cosmological setting and consider only the scalar sector of perturbations, since tensor perturbations' analysis appear to be less contrived. Moreover, we focus on high momenta and frequency modes (as these are the most harmful ones), which means that in all linearized equations we keep only the terms that are the highest order in derivatives. In what follows we adopt the following parametrization for perturbed quantities:

$$
h_{00}=2 \alpha, \quad h_{0 i}=-\partial_{i} \beta, \quad h_{i j}=-a^{2} \cdot 2 \zeta \delta_{i j}, \quad \pi \rightarrow \pi_{0}(t)+\chi,
$$

where $h_{\mu \nu}$ are metric perturbations about a spatially flat FLRW background, while $\chi$ is the Galileon perturbation.

The first step for carrying out the stability analysis is to obtain the quadratic action for perturbations. We discuss two possible approaches, which amount to a specific gauge choice in the scalar sector of perturbations.

The first option is to choose the unitary gauge (i.e. the Galileon perturbation vanishes, $\chi=0$ ), plug the chosen parametrization (2) into the action and expand it to the second order. Upon integrating out $\alpha$ and $\beta$, which turn out to be the non-dynamical degrees of freedom, one obtains the quadratic action for the only scalar degree of freedom left, namely $\zeta$. This approach was adopted independently in Refs. [13, 14] and later generalized to the complete Horndeski theory in Ref. [15]. In what follows, we refer to this approach as KYY for brevity.

Another way to obtain the quadratic action for a cubic Galileon theory was suggested in Ref. [13] (the DPSV approach). This is a bottom up approach, meaning that one reconstructs the quadratic action from the linearized equations of motion. It was noted that, even though the Galileon field equation contains the second derivatives of both metric and Galileon, it is possible to eliminate the second derivatives of metric by making use of Einstein equations. Indeed, the procedure went through for a cubic Galileon theory at a fully non-linear level. Upon linearizing the Galileon equation with the second derivatives of metric having been removed, one can restore the derivative part of the quadratic action with a single degree of freedom, which is $\chi$ in this case. Let us note that one drops the first and zeroth derivatives of both metric and Galileon field in the resulting linearized Galileon equation, having in mind that high momentum modes are of interest only.

Both methods are designed to obtain the derivative part of the quadratic action for scalar perturbations, but the relation between them is not entirely obvious. While the KYY approach chooses the gauge explicitly, the DPSV method seems not to fix one at all. However, this is not entirely so if one looks close enough.

The tricky aspect of the DPSV approach is the fact that in linearized equations of motion one drops all zeroth and first derivatives of metric perturbations, including lapse and shift perturbations ( $\alpha$ and $\partial_{i} \beta$ ). But the analysis of a full quadratic action shows that this might be incorrect. The constraint equations for $\alpha$ and $\partial_{i} \beta$ have the following form:

$$
\begin{gathered}
\alpha=a_{1} \dot{\zeta}+a_{2} \dot{\chi}+\ldots, \\
\vec{\nabla}^{2} \beta=a_{3} \vec{\nabla}^{2} \zeta+a_{4} \vec{\nabla}^{2} \chi+\ldots,
\end{gathered}
$$

where dots denote the terms, which are lower in derivatives, and the coefficients $a_{i}$ stand for combinations of Lagrangian functions and their derivatives. Equations (3) are the variation of a complete quadratic action with respect to $\beta$ and $\alpha$ respectively (we have dropped the common Laplace operator in eq. (3a)). According to the constraint equations (3) $h_{00} \propto \alpha$ should be treated as a first-derivative 
object and $g^{i j} \partial_{i} h_{0 j} \propto \vec{\nabla}^{2} \beta$ should be considered a second-derivative quantity. Hence, the terms like $\dot{\alpha}$ and $\vec{\nabla}^{2} \beta$ should be taken into account, although naively they seem to be first order in derivatives and are supposed to be omitted in the trick.

The most essential observation is that due to gauge invariance of the complete quadratic action and corresponding equations of motion, there are relations between the coefficients ai:

$$
a_{1}=-a_{3}, \quad a_{2}=-a_{4} .
$$

These equalities hold in the most general Horneski theory and imply the relation between the lapse and shift perturbations, which are valid to the leading order in derivatives (i.e. while only the terms with higher derivatives are kept in the constraints (3)):

$$
\alpha=-\dot{\beta}
$$

The relation (5) enables one to choose the comoving gauge $\beta=0$, and automatically obtain $\alpha=0$ (i.e., the gauge turns out to be synchronous as well). Thus, dropping $\dot{\alpha}$ and $\vec{\nabla} \beta$ within the DPSV approach is, in fact, legitimate in Horndeski theory and corresponds to a specific gauge choice. A detailed study of the issue may be found in Ref.[16].

While the KYY method is quite straightforward and widely used for the stability anlysis [17], the DPSV approach simplifies calculations in some cases [18] and may be used as a cross-check of the results obtained in the unitary gauge. Moreover, the DPSV trick was shown to be effective in the study of a wormhole stability [19-21]. These are the reasons for studying the DPSV method in detail.

In this paper we focus on the question whether the DPSV approach works in the most general Horndeski theory (1). We show that unlike the original case, where the trick went through for the unperturbed equations, in complete Horndeski theory the approach works for linearized equations only. The trick also requires a specific choice of both background geometry and background Galileon field. We find that for a spatially flat FLRW background geometry and a homogeneous background Galileon field the generalized DPSV approach gives the correct derivative part of the quadratic action for the scalar degree of freedom. The result for the restored action agrees with the one obtained in the unitary gauge in Ref. [15] modulo an overall factor.

We carry out the generalized DPSV method in detail in the following section.

\section{DPSV trick in the most general Horndeski theory}

Let us consider the DPSV trick in the most general Horndeski theory with the Lagrangian given in (1). The Galileon field equation and Einstein equations have the following form, respectively:

$$
\begin{aligned}
R & {\left[\Omega^{(1)}+\Pi^{(1)}\right]+R^{\mu v}\left[\Omega_{\mu \nu}^{(2)}+\Pi_{\mu \nu}^{(2)}\right]+R_{\mu \nu \lambda \rho}\left[\Omega^{(3) \mu \nu \lambda \rho}+\Pi^{(3) \mu \nu \lambda \rho}\right] } \\
+ & 2 R_{\mu}^{\rho} R_{\nu \rho} \nabla^{\mu} \pi \nabla^{v} \pi G_{5 X}-R_{\mu \nu} R \nabla^{\mu} \pi \nabla^{v} \pi G_{5 X}+2 R^{\rho \sigma} R_{\mu \rho v \sigma} \nabla^{\mu} \pi \nabla^{v} \pi G_{5 X}+\cdots=0 \\
& R\left[\Omega_{\mu \nu}^{(4)}+\Pi_{\mu \nu}^{(4)}\right]+R_{\mu \nu}\left[\Omega^{(5)}+\Pi^{(5)}\right]+R_{\mu}^{\rho}\left[\Omega_{\rho \nu}^{(6)}+\Pi_{\rho \nu}^{(6)}\right]+R_{v}^{\rho}\left[\Omega_{\rho \mu}^{(7)}+\Pi_{\rho \mu}^{(7)}\right] \\
& +R^{\rho \sigma}\left[\Omega_{\mu \nu \rho \sigma}^{(8)}+\Pi_{\mu \nu \rho \sigma}^{(8)}\right]+R_{\mu \rho v \sigma}\left[\Omega^{(9)^{\rho \sigma}}+\Pi^{(9) \rho \sigma}\right]+R_{\mu \rho \sigma \lambda} \Pi_{v}^{(10) \rho \sigma \lambda} \\
& +R_{v \rho \sigma \lambda} \Pi_{\mu}^{(11) \rho \sigma \lambda}+R_{\rho \sigma \lambda \kappa} \Pi_{\mu \nu}^{(12) \rho \sigma \lambda \kappa}+\cdots=0,
\end{aligned}
$$

where dots denote the terms, which do not involve the second derivatives of metric, but do involve second derivatives of the Galileon. In order to avoid bulky equations, we have introduced the objects $\Omega^{(i)}$ 
and $\Pi^{(i)}$, which denote the combinations of Lagrangian functions $F, K, G_{4}, G_{5}$ and their derivatives. The explicit expressions of $\Omega^{(i)}$ and $\Pi^{(i)}$ may be found in Ref. [22].

In full analogy with the original $\mathcal{L}_{3}$ case, the Galileon field equation (6) contains the second derivatives of both metric and Galileon field. The DPSV approach makes use of the Einstein equations (7) in order to get rid of the second derivatives of metric perturbations, so that there is only one scalar degree of freedom left (with second derivatives) in the resulting Galileon equation. It is easy to see, that in eqs. (6) and (7) all the second derivatives of metric got contracted into gravitational tensors. In what follows, we assume that the Ricci tensor and scalar are transformed into the Riemann tensor in both eqs. (6) and (7), which simplifies our argument.

There is a difference in comparison with the $\mathcal{L}_{3}$ case, however. While $\mathcal{L}_{3}$ admitted the trick at the level of unperturbed equations, i.e. one could eliminate the second derivatives of metric in the fully non-linear Galileon equation by using Einstein equations, this is not the case in the most general Horndeski theory. Moreover, upon linearization, eqs. (7) are not enough to get rid of the second derivatives of metric perturbations in the linearized version of eq. (6) in a general background. In other words, without making any assumptions about the background, the second derivative terms in the linearized expression of eq. (6) are not a linear combination of the second derivative terms in the linearized eq.(7). It was checked explicitly by calculating the rank of a relevant matrix with background quantities like $\nabla_{\mu} \pi, \nabla_{\mu} \nabla_{v} \pi$ taking random numerical values. Thus, the DPSV trick does not work in the general Horndeski theory in the same way as it used to in the $\mathcal{L}_{3}$ case.

However, if one assumes that the background geometry is of a flat FLRW type and the background Galileon field is a homogeneous one, i.e. $\pi=\pi(t)$, the trick becomes feasible in the most general Horndeski theory. The only non-zero derivatives of a homogeneous background Galileon are $\dot{\pi}, \ddot{\pi}$ and $\nabla_{i} \nabla_{j} \pi=g_{i j} \dot{\pi} H$, which in turn reduce the number of surviving components of the perturbed Riemann tensor. These are $g^{i j} R_{i 0 j 0}^{\{\mathbf{1}\}}$ and $g^{i j} g^{m n} R_{i m j n}^{\{\mathbf{1}\}}$ (here and below superscript $\{1\}$ denotes linearized quantities).

Under the assumptions made about the background, the linearized Galileon equation reduces to ${ }^{1}$

$$
\begin{aligned}
& g^{i j} R_{i 0 j 0}^{\{\mathbf{1}\}} \cdot\left[-2 G_{4 \pi}+8 G_{4 X} H \dot{\pi}-2 \cdot\left(2 G_{4 \pi X}-K_{X}\right) \dot{\pi}^{2}+16 G_{4 X X} H \dot{\pi}^{3}-4 G_{5 \pi} H \dot{\pi}\right. \\
& \left.+6 G_{5 X} H^{2} \dot{\pi}^{2}+4 G_{5 X X} H^{2} \dot{\pi}^{4}-4 G_{5 \pi X} H \dot{\pi}^{3}\right]+g^{i j} g^{m n} R_{i m j n}^{\{\mathbf{1}\}} \cdot\left[-G_{4 \pi}+2 G_{4 X} H \dot{\pi}\right. \\
& +2 G_{4 X} \ddot{\pi}+2 \dot{\pi}^{2}\left(G_{4 \pi X}+2 G_{4 X X} \ddot{\pi}\right)+G_{5 \pi X} H \dot{\pi}^{3}-G_{5 \pi} H \dot{\pi}-G_{5 \pi} \ddot{\pi}+2 G_{5 X} H \dot{\pi} \ddot{\pi} \\
& \left.+G_{5 X} \dot{\pi}^{2} \frac{\ddot{a}}{a}-G_{5 \pi X} \dot{\pi}^{2} \ddot{\pi}++2 G_{5 X X} H \dot{\pi}^{3} \ddot{\pi}-\frac{1}{2} G_{5 \pi \pi} \dot{\pi}^{2}\right]+Z=0,
\end{aligned}
$$

where $Z$ denotes the terms, which do not contain the second derivatives metric perturbations (we omit the first and zeroth order derivatives of both metric and Galileon perturbations in full analogy with the original trick). Following the DPSV procedure, we need to express the non-zero components of the linearized Riemann tensor $g^{i j} g^{m n} R_{i m j n}^{\{\mathbf{1}\}}$ and $g^{i j} R_{i 0 j 0}^{\{\mathbf{1}\}}$ in (8) through the Galileon perturbations. The same components of the Riemann tensor arise in the $00-$ component and trace $\left(G_{\mu \nu} g^{\mu \nu}\right)^{\{1\}}$ of the linearized Einstein equations:

$$
\begin{aligned}
& g^{i j} g^{m n} R_{i m j n}^{\{\mathbf{1}\}} \cdot\left[2 G_{4 X} \dot{\pi}^{2}-G_{4}-\frac{1}{2} \dot{\pi}^{2}\left(G_{5 \pi}-2 G_{5 X} H \dot{\pi}\right)\right]+Y_{00}=0, \\
& g^{i j} R_{i 0 j 0}^{\{\mathbf{1}\}}\left[4\left(2 G_{4 X} \dot{\pi}^{2}-G_{4}\right)-2 \dot{\pi}^{2}\left(G_{5 \pi}-2 G_{5 X} H \dot{\pi}\right)\right] \\
+ & g^{i j} g^{m n} R_{i m j n}^{\{\mathbf{1}\}}\left[2\left(G_{4 X} \dot{\pi}^{2}-G_{4}\right)+G_{5 X} \dot{\pi}^{2}(H \dot{\pi}+\ddot{\pi})\right]+g^{\mu \nu} Y_{\mu \nu}=0,
\end{aligned}
$$

\footnotetext{
${ }^{1}$ In what follows Latin indices take values $1,2,3$.
} 
where $Y_{00}$ and $g^{\mu \nu} Y_{\mu \nu}$ again involve the second derivatives of the Galileon perturbation, but not metric perturbations.

As was explained above, the subtlety with the lapse and shift perturbations can be safely ignored, since in the general Horndeski case a comoving gauge is automatically the synchronous one. Hence, we impose this gauge from the very beginning and safely drop both $\dot{\alpha}$ and $\vec{\nabla} \beta$.

In order to obtain the equation that is free of the second derivatives of metric perturbations, we make use of eqs. (9a) and (9b) and express the structures $g^{i j} g^{m n} R_{i m j n}^{\{\mathbf{1}\}}$ and $g^{i j} R_{i 0 j 0}^{\{\mathbf{1}\}}$ in terms of the Galileon perturbation. Upon substituting them into eq. (8) we obtain the following equation:

$$
\begin{aligned}
& {\left[Y_{00}\left(2 G_{4 X} \dot{\pi}^{2}-2 G_{4}+G_{5 X} \dot{\pi}^{2}(H \dot{\pi}+\ddot{\pi})\right)-g^{\mu \nu} Y_{\mu \nu}\left(2 G_{4 X} \dot{\pi}^{2}-G_{4}-\frac{1}{2} \dot{\pi}^{2}\left(G_{5 \pi}-2 G_{5 X} H \dot{\pi}\right)\right)\right]} \\
& \times\left(-2 G_{4 \pi}+8 G_{4 X} H \dot{\pi}-2 \cdot\left(2 G_{4 \pi X}-K_{X}\right) \dot{\pi}^{2}+16 G_{4 X X} H \dot{\pi}^{3}-4 G_{5 \pi} H \dot{\pi}+6 G_{5 X} H^{2} \dot{\pi}^{2}\right. \\
& \left.+4 G_{5 X X} H^{2} \dot{\pi}^{4}-4 G_{5 \pi X} H \dot{\pi}^{3}\right)-Y_{00}\left[4 G_{4 X} \dot{\pi}^{2}-2 G_{4}-\dot{\pi}^{2}\left(G_{5 \pi}-2 G_{5 X} H \dot{\pi}\right)\right] \\
& \times\left(-2 G_{4 \pi}+4 G_{4 X} H \dot{\pi}+4 G_{4 X} \ddot{\pi}+4 \dot{\pi}^{2}\left(G_{4 \pi X}+2 G_{4 X X} \ddot{\pi}\right)+2 G_{5 \pi X} H \dot{\pi}^{3}-2 G_{5 \pi} H \dot{\pi}-2 G_{5 \pi} \ddot{\pi}\right. \\
& \left.+4 G_{5 X} H \dot{\pi} \ddot{\pi}+2 G_{5 X} \dot{\pi}^{2} \frac{\ddot{a}}{a}-2 G_{5 \pi X} \dot{\pi}^{2} \ddot{\pi}+4 G_{5 X X} H \dot{\pi}^{3} \ddot{\pi}-G_{5 \pi \pi} \dot{\pi}^{2}\right) \\
& +Z\left(4 G_{4 X} \dot{\pi}^{2}-2 G_{4}-\dot{\pi}^{2}\left(G_{5 \pi}-2 G_{5 X} H \dot{\pi}\right)\right)^{2}=0 .
\end{aligned}
$$

Let us recall that $Y_{00}, Y_{\mu \nu}$ and $Z$ do not contain the second derivatives of metric perturbations and, hence, the only scalar degree of freedom left in the linearized equation (10) is $\chi$.

The final step is to reconstruct the quadratic action from the obtained equation (10) for $\chi$. Taking eq. (10) as a result of variation with respect to $\chi$ of a quadratic action, one may reconstruct the latter:

$$
S_{\text {gal }}^{(2)}=\int \mathrm{d} t \mathrm{~d}^{3} x a^{3}\left[\tilde{\mathcal{A}} \dot{\chi}^{2}-\tilde{\mathcal{B}} \frac{(\vec{\nabla} \chi)^{2}}{a^{2}}\right],
$$

where coefficients $\tilde{\mathcal{A}}$ and $\tilde{\mathcal{B}}$ have the following form

$$
\tilde{\mathcal{A}}=\frac{\Sigma \mathcal{G}_{\mathcal{T}}+3 \Theta^{2}}{\mathcal{G}_{\mathcal{T}} \dot{\pi}^{2}}, \quad \tilde{\mathcal{B}}=\frac{\Theta^{2}}{\mathcal{G}_{\mathcal{T}}^{2} \dot{\pi}^{2}}\left(\frac{1}{a} \frac{\mathrm{d}}{\mathrm{d} t}\left[\frac{a \mathcal{G}_{\mathcal{T}}{ }^{2}}{\Theta}\right]-\mathcal{F}_{\mathcal{T}}\right),
$$

and

$$
\begin{aligned}
& \mathcal{G}_{\mathcal{T}}=2 G_{4}-4 G_{4 X} \dot{\pi}^{2}+G_{5 \pi} \dot{\pi}^{2}-2 H G_{5 X} \dot{\pi}^{3} \\
& \mathcal{F}_{\mathcal{T}}=2 G_{4}-2 G_{5 X} \dot{\pi}^{2} \ddot{\pi}-G_{5 \pi} \dot{\pi}^{2} \\
& \Theta=-K_{X} \dot{\pi}^{3}+2 G_{4} H-8 H G_{4 X} \dot{\pi}^{2}-8 H G_{4 X X} \dot{\pi}^{4}+G_{4 \pi} \dot{\pi}+2 G_{4 \pi X} \dot{\pi}^{3}- \\
& -5 H^{2} G_{5 X} \dot{\pi}^{3}-2 H^{2} G_{5 X X} \dot{\pi}^{5}+3 H G_{5 \pi} \dot{\pi}^{2}+2 H G_{5 \pi X} \dot{\pi}^{4} \\
& \Sigma=F_{X} \dot{\pi}^{2}+2 F_{X X} \dot{\pi}^{4}+12 H K_{X} \dot{\pi}^{3}+6 H K_{X X} \dot{\pi}^{5}-K_{\pi} \dot{\pi}^{2}-K_{\pi X} \dot{\pi}^{4}- \\
& -6 H^{2} G_{4}+42 H^{2} G_{4 X} \dot{\pi}^{2}+96 H^{2} G_{4 X X} \dot{\pi}^{4}+24 H^{2} G_{4 X X X} \dot{\pi}^{6}- \\
& -6 H G_{4 \pi} \dot{\pi}-30 H G_{4 \pi X} \dot{\pi}^{3}-12 H G_{4 \pi X X} \dot{\pi}^{5}+30 H^{3} G_{5 X} \dot{\pi}^{3}+ \\
& +26 H^{3} G_{5 X X} \dot{\pi}^{5}+4 H^{3} G_{5 X X X} \dot{\pi}^{7}-18 H^{2} G_{5 \pi} \dot{\pi}^{2}-27 H^{2} G_{5 \pi X} \dot{\pi}^{4}- \\
& -6 H^{2} G_{5 \pi X X} \dot{\pi}^{6} .
\end{aligned}
$$


Note that the procedure above enables us to reconstruct only the derivative part of the quadratic action, since we have dropped the zeroth and first derivatives of both $\chi$ and metric perturbations. The coefficients $\Theta, \Sigma, G_{\mathcal{T}}$ and $\mathcal{F}_{\mathcal{T}}$ coincide with the expressions given in Ref. [15]. Modulo an overall positive factor $\Theta^{-2} \mathcal{G}_{\mathcal{T}}{ }^{2} \dot{\pi}^{2}$ in the integrand, the action (11) coincide with that found in Ref. [15] in the unitary gauge. This shows that modulo field redefinition, the DPSV approach leads to the correct quadratic action at the derivative level for the only scalar degree of freedom. This completes the analysis of the DPSV approach in the most general Horndeski theory.

\section{Acknowledgements}

The author is indebted to the collaborators R. Kolevatov, S. Mironov, V. Rubakov and N. Sukhov. This work is supported by the Foundation for the Advancement of Theoretical Physics and Mathematics "BASIS" grant.

\section{References}

[1] G. W. Horndeski, Int. J. Theor. Phys. 10, 363 (1974)

[2] V. A. Rubakov, Phys. Usp. 57, 128-142, 2014, ,Usp. Fiz. Nauk 184 no.2, 137-152 (2014)

[3] D. A. Easson, I. Sawicki and A. Vikman, JCAP 1111021 (2011)

[4] Y. F. Cai, D. A. Easson and R. Brandenberger, JCAP 1208020 (2012)

[5] M. Koehn, J. L. Lehners and B. A. Ovrut, Phys. Rev. D 90 no.2, 025005 (2014)

[6] L. Battarra, M. Koehn, J. L. Lehners and B. A. Ovrut, JCAP 1407007 (2014)

[7] T. Qiu and Y. T. Wang, JHEP 1504130 (2015)

[8] T. Kobayashi, M. Yamaguchi and J. Yokoyama, JCAP 1507 no.07, 017 (2015)

[9] Y. Wan, T. Qiu, F. P. Huang, Y. F. Cai, H. Li and X. Zhang, JCAP 1512 no.12, 019 (2015)

[10] A. Ijjas and P. J. Steinhardt, Phys. Rev. Lett. 117 no.12, 121304 (2016)

[11] Y. Cai, Y. Wan, H. G. Li, T. Qiu and Y. S. Piao, JHEP 1701090 (2017)

[12] P. Creminelli, D. Pirtskhalava, L. Santoni and E. Trincherini, JCAP 1611 no.11, 047 (2016)

[13] C. Deffayet, O. Pujolas, I. Sawicki and A. Vikman, JCAP 1010026 (2010)

[14] T. Kobayashi, M. Yamaguchi and J. Yokoyama, Phys. Rev. Lett. 105231302 (2010)

[15] T. Kobayashi, M. Yamaguchi and J. Yokoyama, Prog. Theor. Phys. 126511 (2011)

[16] R. Kolevatov, S. Mironov, V. Rubakov, N. Sukhov and V. Volkova, Phys. Rev. D 96 no.12, 125012 (2017)

[17] T. Kobayashi, Phys. Rev. D 94, no. 4, 043511 (2016)

[18] M. Libanov, S. Mironov and V. Rubakov, JCAP 1608, no. 08, 037 (2016)

[19] V. A. Rubakov, Theor. Math. Phys. 188, no. 2, 1253 (2016)

[20] R. Kolevatov and S. Mironov, Phys. Rev. D 94 no.12, 123516 (2016)

[21] O. A. Evseev and O. I. Melichev, Phys. Rev. D 96 no.2, 024030 (2017)

[22] S. Mironov and V. Volkova, Preprint: arXiv:1712.09909 [hep-th]. 